\title{
Inferring magnetic helicity spectrum in spherical domains: Method and example applications
}

\author{
A. P. Prabhu ${ }^{1}$, N. K. Singh ${ }^{2}$, M. J. Käpylä ${ }^{3,1,4}$, and A. $\operatorname{Lagg}^{1,3}$ \\ 1 Max Planck Institute for Solar System Research, Justus-von-Liebig-Weg 3, 37077 Göttingen, Germany \\ e-mail: prabhu@mps.mpg.de \\ 2 Inter-University Centre for Astronomy and Astrophysics, Post Bag 4, Ganeshkind, Pune 411007, India \\ 3 Department of Computer Science, Aalto University, PO Box 15400, 00076 Aalto, Finland \\ ${ }^{4}$ NORDITA, KTH Royal Institute of Technology and Stockholm University, Hannes Alfvéns väg 12, 11419 Stockholm, Sweden
}

Received 15 April 2021 / Accepted 8 July 2021

\section{ABSTRACT}

\begin{abstract}
Context. Obtaining observational constraints on the role of turbulent effects for the solar dynamo is a difficult, yet crucial, task. Without such knowledge, the full picture of the operation mechanism of the solar dynamo cannot be formed.

Aims. The magnetic helicity spectrum provides important information about the $\alpha$ effect. Here we demonstrate a formalism in spherical geometry to infer magnetic helicity spectra directly from observations of the magnetic field, taking into account the sign change of magnetic helicity across the Sun's equator.

Methods. Using an angular correlation function of the magnetic field, we develop a method to infer spectra for magnetic energy and helicity. The retrieval of the latter relies on a fundamental definition of helicity in terms of linkage of magnetic flux. We apply the two-scale approach, previously used in Cartesian geometry, to spherical geometry for systems where a sign reversal of helicity is expected across the equator on both small and large scales.

Results. We test the method by applying it to an analytical model of a fully helical field, and to magneto-hydrodynamic simulations of a turbulent dynamo. The helicity spectra computed from the vector potential available in the models are in excellent agreement with the spectra computed solely from the magnetic field using our method. In a next test, we use our method to obtain the helicity spectrum from a synoptic magnetic field map corresponding to a Carrington rotation. We observe clear signs of a bihelical spectrum of magnetic helicity, which is in complete accordance to the previously reported spectra in literature from the same map.

Conclusions. Our formalism makes it possible to infer magnetic helicity in spherical geometry, without the necessity of computing the magnetic vector potential. It has many applications in solar and stellar observations, but can also be used to analyse global magnetoconvection models of stars and to compare them with observations.
\end{abstract}

Key words. Sun: magnetic fields - magnetohydrodynamics (MHD) - dynamo - turbulence

\section{Introduction}

The solenoidal nature of magnetic fields enables us to examine them in terms of the topology of closed curves (Berger \& Field 1984). Helicity integrals in general, and magnetic helicity in particular, (see Eq. (1)) have been demonstrated to be associated with the topological properties of field lines (Moffatt 1969, 1978). Magnetic helicity, which characterises the linkage of field lines, is a topological invariant. In ideal magnetohydrodynamics (MHD) magnetic helicity is conserved (Woltjer 1958), and is nearly conserved in the limit of large conductivity (Berger 1984). Thus, magnetic helicity imposes a crucial constraint on the evolution of magnetic fields.

In the case of the Sun, magnetic helicity is often invoked to investigate many facets of the solar magnetic field. To name a few examples, it has been suggested as a possible proxy to quantify the eruptivity of solar active regions (ARs; Pariat et al. 2017; Thalmann et al. 2019), or as a plausible predictor of the solar cycle (Hawkes \& Berger 2018). The influence of magnetic helicity on coronal emission, to explain the observed enhancement in X-ray luminosity with increasing stellar rotation, has also been explored (Warnecke \& Peter 2019). Arguably, magnetic helicity plays the most crucial part in dynamo theory, which is often called upon to explain the generation and maintenance of the solar magnetic field (Moffatt 1978;
Brandenburg \& Subramanian 2005; Brandenburg 2018; Rincon 2019). Specifically the $\alpha$ effect, occurring in systems with stratification and rotation, is expected to be an important inductive effect in the solar dynamo. Under isotropic and homogeneous conditions $\alpha$ is known to be related to the kinetic helicity of the flow and is a measure of the helical nature of turbulence within the Sun's convection zone. It has been shown that the $\alpha$ effect generates bihelical magnetic fields, that is, magnetic helicity at small and large scales of opposite signs, hence resulting in no net production of magnetic helicity (Seehafer 1996; Ji 1999; Yousef \& Brandenburg 2003). For the Sun, another sign change of magnetic helicity is expected across the equator, due to the combined action of stratification and the Coriolis force breaking reflectional symmetry. Thus we have a hemispheric sign rule (HSR, see Singh et al. 2018, for details). The HSR implies a positive (negative) sign of magnetic helicity at large (small) scales in the northern hemisphere and vice versa in southern hemisphere. Observational evidence for such a sign rule will be an indirect confirmation of the role played by the $\alpha$ effect in generating large-scale solar magnetic fields.

Magnetic helicity is defined in terms of $\boldsymbol{A}$, the magnetic vector potential and the magnetic field, $\boldsymbol{B}$, as

$\mathcal{H}_{\mathrm{M}}=\int \boldsymbol{A} \cdot(\boldsymbol{\nabla} \times \boldsymbol{A}) \mathrm{d}^{3} x=\int \boldsymbol{A} \cdot \boldsymbol{B} \mathrm{d}^{3} x$ 
The first hurdle in inferring magnetic helicity observationally arises due to the fact that $\boldsymbol{A}$ in Eq. (1) is not an observed quantity and secondly, the information about $\boldsymbol{B}$ is usually not known over the entire volume. Given these restrictions, prior efforts to study the magnetic helicity of ARs used current helicity $\mathcal{H}_{\mathrm{C}}=\langle\boldsymbol{J} \cdot \boldsymbol{B}\rangle_{\mathrm{V}}$ as a proxy, where $\boldsymbol{J}$ is the current density and the angle brackets denote volume averages. Additionally the simplifying assumption of a force-free magnetic field is made (Seehafer 1990; Pevtsov et al. 1995; Bao et al. 1999; Zhang et al. 2010). If in Eq. (1) $\boldsymbol{B}$ has a non-zero normal component at the boundary of the integration volume, then the volume integral cannot be determined in a gauge-invariant manner. This is true for most astrophysical systems including the Sun. For such situations the concept of relative magnetic helicity can prove useful (Berger \& Field 1984; Finn \& Antonsen 1985): in this approach, helicity is defined with respect to a reference field, usually a current-free one. Relative magnetic helicity is often used to analyse solar observations, as is demonstrated in a recent study by Thalmann et al. (2019). Using nonlinear forcefree field extrapolations as input, these approaches involve an explicit computation of $\boldsymbol{A}$ making suitable gauge choices. For a review of these and other related methods see Valori et al. (2016).

Using a toroidal-poloidal decomposition (Chandrasekhar 1961) of vector fields in spherical geometry, Pipin et al. (2019) compute the magnetic helicity from solar synoptic maps by reconstructing $\boldsymbol{A}$, again making a particular gauge choice. In their analysis they separate the large and small scales relying on azimuthal averages, wherein the small-scale magnetic helicity density includes contributions from all scales except the axisymmetric mean field, including the large-scale non-axisymmetric contributions. Lund et al. (2020) also resort to such a decomposition, applying it on stellar data retrieved via Zeeman-Doppler imaging (ZDI, Semel 1989), although they report large-scale average magnetic helicity density on stellar surfaces. However, in these methods, the total value of magnetic helicity density does not provide us the distribution of helicity, specifically its sign, over scales.

To study the dynamo problem, for which the segregation of helicity over spatial scales is expected, methods focused on inferring spectral distribution of magnetic helicity from observations are better suited. Zhang et al. (2014, 2016) inferred such a spectrum of magnetic helicity from local patches, invoking the two-point correlation tensor for magnetic field in Cartesian geometry. Brandenburg et al. (2017) extended this approach to inhomogeneous systems by using the two-scale analysis of Roberts \& Soward (1975), applicable to cases where magnetic helicity varies slowly, on a scale somewhat smaller than the scale of the system. It is important to note that these methods avoid any issues with a gauge choice by transforming into Fourier space with the implicit assumption of periodicity. Recently Prior et al. (2020) demonstrated a wavelet based multi-resolution analysis to infer magnetic helicity which is applicable for inhomogeneous systems with non-periodic boundaries. They also avoid computing the vector potential and thus avoid making a gauge choice by relying on a topologically meaningful definition of helicity in terms of $\boldsymbol{B}$. The investigation of the helicity distribution on global scales, i.e., over the whole surface of the Sun or other stars, requires the use of a spherical geometry. To investigate the helicity distribution over scales on the surface of the Sun or other stars, the observations are available in spherical geometry. In this context Brandenburg et al. (2017) regarded their Cartesian approach as preliminary and highlighted the need to do an analogous analysis in spherical harmonics. Prior et al. (2020) also assume a Cartesian volume for their multi-resolution wavelet decomposition.

In this paper we rely on a similar topological definition of helicity in terms of linking of $\boldsymbol{B}$, and adapt it to spherical geometry by using spherical harmonics. Here we extend the Cartesian approach of Brandenburg et al. (2017) and do a treatment of scales based on spherical harmonic degree in contrast to the approach of Pipin et al. (2019) which is based on azimuthal averaging. Our method is suited for solar and stellar observations, where photospheric magnetic field observations are available in spherical geometry via spectropolarimetric inversions or ZDI. Particularly for the Sun, it also enables access to higher cadence full disk magnetogram data. It is also readily applicable to solar and stellar dynamo models, where the induction equation is typically expressed in $\boldsymbol{B}$ and numerically integrated, to compute magnetic helicity from such models and compare them with observations, where $\boldsymbol{A}$ is not available.

This paper is organised as follows: In Sect. 2 we describe our present method for spherical geometry in the context of the existing Cartesian framework. In Sect. 3, we test this formalism against an analytical expression of a magnetic field on a spherical shell. Moreover, we also apply it to a three-dimensional turbulent dynamo simulation in spherical geometry. Finally we also apply a simple modification of our approach to synoptic maps of the Sun and compare it with previously obtained results. We discuss the scope of applicability of our formalism to observations in Sect. 4.

\section{The method and formalism}

\subsection{Definitions}

We begin by defining the spectra for magnetic energy and helicity on a spherical shell. In such situations it is convenient to use spherical harmonics, $Y_{\ell}^{m}(\theta, \phi)$, where $\ell$ represents the degree and $m$ the azimuthal order. Thus we can define the spectra of magnetic energy as,

$2 E_{\mathrm{M}}(\ell)=\sum_{m=-\ell}^{\ell} b_{i}^{\ell m} b_{i}^{\ell m *}$,

where $b_{i}^{\ell m}=\int B_{i}(\theta, \phi) Y_{\ell}^{m}(\theta, \phi) \mathrm{d} \Omega$ is the expansion coefficient of the $i$ th component of $\boldsymbol{B}, i=(r, \theta, \phi)$, and the star symbol (*) denotes the complex conjugate of the coefficient. On such a spherical shell we have $\int \mathrm{d} \Omega=4 \pi$ as the surface area of a unit sphere. Analogously, following Eq. (1), we can define a spectrum of magnetic helicity as

$H_{\mathrm{M}}(\ell)=\sum_{m=-\ell}^{\ell} \frac{1}{2}\left[a_{i}^{\ell m *} b_{i}^{\ell m}+a_{i}^{\ell m} b_{i}^{\ell m *}\right]$.

These spectra can be directly related to the values of magnetic energy and helicity using Plancherel's theorem:

$$
\begin{aligned}
& \mathcal{E}_{\mathrm{M}} \equiv \frac{1}{4 \pi} \int \frac{1}{2} \boldsymbol{B}^{2} \mathrm{~d} \Omega=\sum_{\ell=0}^{\infty} E_{\mathrm{M}}(\ell), \quad \text { and } \\
& \mathcal{H}_{\mathrm{M}} \equiv \frac{1}{4 \pi} \int \boldsymbol{A} \cdot \boldsymbol{B} \mathrm{d} \Omega=\sum_{\ell=0}^{\infty} H_{\mathrm{M}}(\ell) .
\end{aligned}
$$

As has been demonstrated in Moffatt (1969) and Berger \& Field (1984), for a given field $\boldsymbol{B}$, we can define 
the magnetic helicity in terms of the linkage of its flux, thus using the Gauss linking formula:

$\mathcal{H}_{\mathrm{M}}=\frac{1}{4 \pi} \iint \boldsymbol{B}(\boldsymbol{x}) \cdot\left[\boldsymbol{B}(\boldsymbol{y}) \times \frac{\boldsymbol{x}-\boldsymbol{y}}{|\boldsymbol{x}-\boldsymbol{y}|^{3}}\right] \mathrm{d}^{3} x \mathrm{~d}^{3} y$.

This definition is equivalent to that of Eq. (1) if one adopts the Coulomb gauge, $\boldsymbol{\nabla} \cdot \boldsymbol{A}=0$, and uses the Biot-Savart law (Moffatt \& Ricca 1992; Subramanian \& Brandenburg 2006). Subramanian \& Brandenburg (2006) demonstrate how this definition allows for a gauge-invariant, physically meaningful description of magnetic helicity density.

\subsection{Cartesian geometry}

(a) Homogeneous case. In this section we first reiterate the approach delineated in Moffatt (1978) that relates the correlation function of the magnetic field to its energy and helicity in homogeneous conditions. The magnetic energy spectrum, $E_{\mathrm{M}}(k)$, is obtained from $2 E_{\mathrm{M}}(k)=\int \delta_{i j} \hat{M}_{i j}(\boldsymbol{k}) k \mathrm{~d} \Omega$, where $\hat{M}_{i j}(\boldsymbol{k})$ is the 2D Fourier transform of the two-point correlation tensor of the total magnetic field, i.e., $M_{i j}(\boldsymbol{\xi})=\left\langle B_{i}(\boldsymbol{x}) B_{j}(\boldsymbol{x}+\boldsymbol{\xi})\right\rangle$, which depends on the separation $\xi$ between the two points. This tensor is assumed to be independent of the position vector $\boldsymbol{x}$ under homogeneous conditions; the brackets denote an ensemble average and $\boldsymbol{k}$ is the conjugate variable to $\boldsymbol{x}$ spanning the 2D Cartesian surface. The scaled magnetic helicity spectrum, $k H_{\mathrm{M}}(k)$, with same dimensions as that of $E_{\mathrm{M}}(k)$, is defined as $k H_{\mathrm{M}}(k)=\int \mathrm{i} \hat{k}_{i} \epsilon_{i j k} \hat{M}_{j k}(\boldsymbol{k}) k \mathrm{~d} \Omega$, with $\hat{k}_{i}=k_{i} /|\boldsymbol{k}|$ being the unit vector of $\boldsymbol{k}$.

(b) Inhomogeneous case. This formalism was extended to inhomogeneous conditions in Brandenburg et al. (2017). Under such conditions, for a given magnetic field $\boldsymbol{B}(\boldsymbol{x}, t)$, with $\boldsymbol{x}$ being the position vector at a time $t$, its two-point correlation tensor in Fourier space takes the form of

$\tilde{M}_{i j}(\boldsymbol{K}, \boldsymbol{k})=\left\langle\hat{B}_{i}\left(\boldsymbol{k}+\frac{1}{2} \boldsymbol{K}\right) \hat{B}_{j}^{*}\left(\boldsymbol{k}-\frac{1}{2} \boldsymbol{K}\right)\right\rangle$.

Here $\boldsymbol{K}$ is the conjugate variable to $\boldsymbol{X}=\left(\boldsymbol{x}^{\prime}+\boldsymbol{x}^{\prime \prime}\right) / 2$, the slowly varying coordinate, and $\boldsymbol{k}$ the conjugate to $\boldsymbol{x}=\boldsymbol{x}^{\prime}-$ $\boldsymbol{x}^{\prime \prime}$, the distance between the two points around $\boldsymbol{X}$. For details see Roberts \& Soward (1975), Brandenburg et al. (2017). For brevity we henceforth omit specifying explicitly the time dependence. Then the spectrum for magnetic energy and helicity are given by

$2 \tilde{E}_{\mathrm{M}}(\boldsymbol{K}, k)=\int \delta_{i j} \tilde{M}_{i j}(\boldsymbol{K}, \boldsymbol{k}) k \mathrm{~d} \Omega$,

$k \tilde{H}_{\mathrm{M}}(\boldsymbol{K}, k)=\int i \hat{k}_{i} \epsilon_{i j k} \tilde{M}_{j k}(\boldsymbol{K}, \boldsymbol{k}) k \mathrm{~d} \Omega$,

where $\int \mathrm{d} \Omega=2 \pi$. Therefore, the trace of $\tilde{M}_{i j}$ gives the magnetic energy and its skew-symmetric part the magnetic helicity. Equation (9) is related to the definition of helicity in Eq. (6). The spectrum is thus computed by the product of magnetic fields that are shifted by a wavenumber corresponding to the large-scale modulation of the slowly varying coordinate. We will draw upon this idea in Sect. 3.2.

\subsection{Spherical geometry}

(a) Homogeneous case. For a quantity distributed on a sphere, the use of an angular correlation function to extract its power spectrum has been demonstrated by Peebles (1973) in the context of cosmology. Here we apply these principles to an angular correlation tensor of the total magnetic field vector on the observed surface of a star. For the magnetic field vector at two positions $\boldsymbol{\Omega}_{1}$ and $\boldsymbol{\Omega}_{2}$ on a unit sphere, the correlation function is of the form (see Appendix A for details),

$M_{i j}(\chi)=\int \frac{1}{8 \pi^{2}} B_{i}\left(\mathbf{\Omega}_{1}\right) B_{j}\left(\mathbf{\Omega}_{2}\right) \delta\left(\cos \chi_{12}-\cos \chi\right) \mathrm{d} \Omega_{1} \mathrm{~d} \Omega_{2}$,

with $\boldsymbol{\Omega}=(\theta, \phi)$, and $\theta$ and $\phi$ are the colatitude and azimuth respectively and $\chi_{12}$ is the angle between the two directions $\boldsymbol{\Omega}_{1}$ and $\boldsymbol{\Omega}_{2}$. Here we assume homogeneity, where $M_{i j}$ is assumed to depend only on the separation $\chi$. The delta function ensures the product is evaluated at the angular separation and the denominator is needed for normalisation (Peebles 1973). Now, analogous to the Cartesian case, the spectrum of magnetic energy is given by the trace of the correlation tensor as (see Appendix B)

$2 E_{\mathrm{M}}(\ell)=2 \pi(2 \ell+1) \int \delta_{i j} M_{i j}(\chi) P_{\ell}(\cos \chi) \mathrm{d} \cos \chi$,

and the scaled spectrum of magnetic helicity is given by its skewsymmetric part (see Appendix C),

$k H_{\mathrm{M}}(\ell)=2 \pi \int \epsilon_{i j k} \nabla_{i}^{\prime}\left(P_{\ell}(\cos \chi) M_{j k}(\chi) \mathrm{d} \cos \chi\right.$.

Here, $\nabla^{\prime} \equiv \partial / \partial \theta_{2}+\left(1 / \sin \theta_{2}\right) \partial / \partial \phi_{2}$ acts on location $\boldsymbol{\Omega}_{2}\left(\theta_{2}, \phi_{2}\right)$, and $\chi=\chi_{12}$ is the angular separation between $\boldsymbol{\Omega}_{1}$ and $\boldsymbol{\Omega}_{2}$; see Appendix $C$ for a derivation and note that $k R \approx \ell+1 / 2$ by the Jeans relation where $R$, taken here as unity, is the radius of the sphere. We use the general expression given in Eq. (C.4), which directly determines the linkages of magnetic field lines to yield the magnetic helicity, and is also applicable to the inhomogeneous case discussed below. Equation (12), as written above, may be obtained by first taking the ensemble average of Eq. (C.4) and then using the homogeneous form of $M_{i j}(\chi)$ given in Eq. (10).

(b) Inhomogeneous case. We focus here on slow latitudinal variation of magnetic helicity which has opposite signs in the two hemispheres; see Singh et al. (2018) for the expected HSR of the Sun. For the magnetic energy spectrum, we continue to use the homogeneous formulation, specifically Eqs. (10) and (11), described above. As noted in Appendix C, the two-point correlation function $M_{i j}(\boldsymbol{\Omega}, \chi)$ depends on both the position on the sphere as well as the separation between the two points; $\boldsymbol{\Omega}$ here represents a mean location that lies between the two points. We note that the formulation in Appendix $\mathrm{C}$ yields a general expression for the magnetic helicity, based on the Gauss linking formula, without making any assumption of homogeneity. We make use of Eqs. (C.4) and (C.6) to determine the SMHS in both the homogeneous, with $M_{i j}=M_{i j}(\chi)$, and the inhomogeneous, with $M_{i j}=M_{i j}(\boldsymbol{\Omega}, \chi)$, cases. Thus, it is the form of the correlation function $M_{i j}$ that allows us to also explore the inhomogeneous cases.

In exact analogy to the Cartesian case discussed earlier in Sect. 2.2, we find that the inhomogeneous case corresponds simply to a shift in the spherical degree $\ell$ by, say, $\mathcal{L}$, while determining the spherical transform of $M_{i j}$; see Eqs. (7) and (9) for the analogy, and Brandenburg (2019) where this generalisation in the spherical domain was discussed. The scheme to determine the helicity in this case may be briefly sketched here as: $H_{\mathrm{M}}(\mathcal{L}, \ell) \propto\left\langle b_{i}^{\ell m} b_{j}^{* \ell+\mathcal{L}, m}\right\rangle$, where $b_{i}^{\ell m}$ and $b_{j}^{* \ell+\mathcal{L}, m}$ correspond to the two components of the magnetic field. We have chosen $\mathcal{L}=1$ 


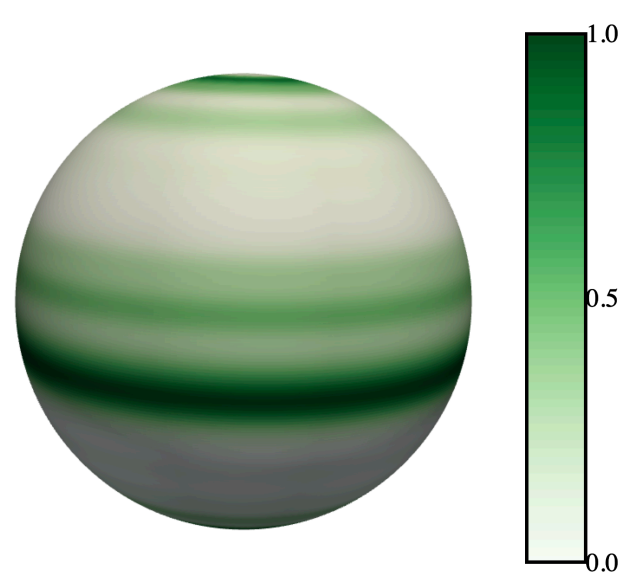

Fig. 1. Distribution of magnetic helicity density obtained from $\boldsymbol{A}$, defined in Eq. (13), and $\boldsymbol{B}=\alpha \boldsymbol{A}$. The color represents its magnitude which has been normalised by the maximum value. This figure was made using PyVista (Sullivan \& Kaszynski 2019). The grey tinge is simply a shading effect.

in the present study where it corresponds to the large scale modulation of the magnetic helicity; as $P_{1}(\cos \theta)=\cos \theta$, it naturally involves a sign change across the equator where $\theta=\pi / 2$. To show the spectrum of magnetic helicity in the sections below, we adopt a sign convention that corresponds to the northern hemisphere, where the large-scale fields have positive helicity.

\section{Testing the method}

To test whether our formalism allows us to infer the spatial distribution magnetic helicity over a sphere relying only on $\boldsymbol{B}$, we subject it to cases where we know both $\boldsymbol{A}$ and $\boldsymbol{B}$. In Sect. 3.1, we use an analytical expression of a fully helical magnetic field, and in Sect. 3.2 a simulated magnetic field generated by a turbulent dynamo. Finally, we apply it to synoptic vector magnetograms of the Sun in Sect. 3.3.

\subsection{Fully helical field}

Chandrasekhar \& Kendall (1957) derived general solutions of the equation $\boldsymbol{\nabla} \times \boldsymbol{B}=\alpha \boldsymbol{B}$, in the context of force-free magnetic fields for spherical geometry commonly known as Chandrasekhar-Kendall functions. Here we use these functions, $\boldsymbol{T}=\boldsymbol{\nabla} \times(\phi \hat{r})$ and $\boldsymbol{S}=\boldsymbol{\nabla} \times \boldsymbol{T} / \alpha$, to define the vector potential on a unit sphere as $\boldsymbol{A}=\boldsymbol{T}+\boldsymbol{S}$. We expand $\phi$ in spherical harmonics as $\phi=\sum_{\ell m} c^{\ell m} Y_{\ell}^{m}(\mathbf{\Omega})$, assume axisymmetry and ignore the radial dependence of the coefficients $c^{\ell m}$ while choosing their values in a random fashion. Thus we have the vector potential as,

$$
\begin{aligned}
\boldsymbol{A}(\boldsymbol{\Omega}) & =\frac{-1}{\alpha} L^{2} \phi \hat{\boldsymbol{r}}-\frac{\partial \phi}{\partial \theta} \hat{\boldsymbol{\phi}} \\
& =\frac{1}{\alpha} \sum_{\ell m} \ell(\ell+1) c^{\ell m} Y_{\ell}^{m}(\theta, \phi) \hat{\boldsymbol{r}}-\sum_{\ell m} c^{\ell m} \frac{\partial}{\partial \theta} Y_{\ell}^{m}(\theta, \phi) \hat{\boldsymbol{\phi}}
\end{aligned}
$$

Here, $L^{2}$ is the angular part of the Laplace operator in spherical geometry such that $L^{2} Y_{\ell}^{m}=-\ell(\ell+1) Y_{\ell}^{m}$ and $\alpha=\sqrt{\ell(\ell+1)}$. From this the magnetic field follows as $\boldsymbol{B}=\boldsymbol{\nabla} \times \boldsymbol{A}=\alpha(\boldsymbol{T}+\boldsymbol{S})$. To evaluate this numerically we use the Python interface of SHTOOLS (Wieczorek \& Meschede 2018). Figure 1 shows the

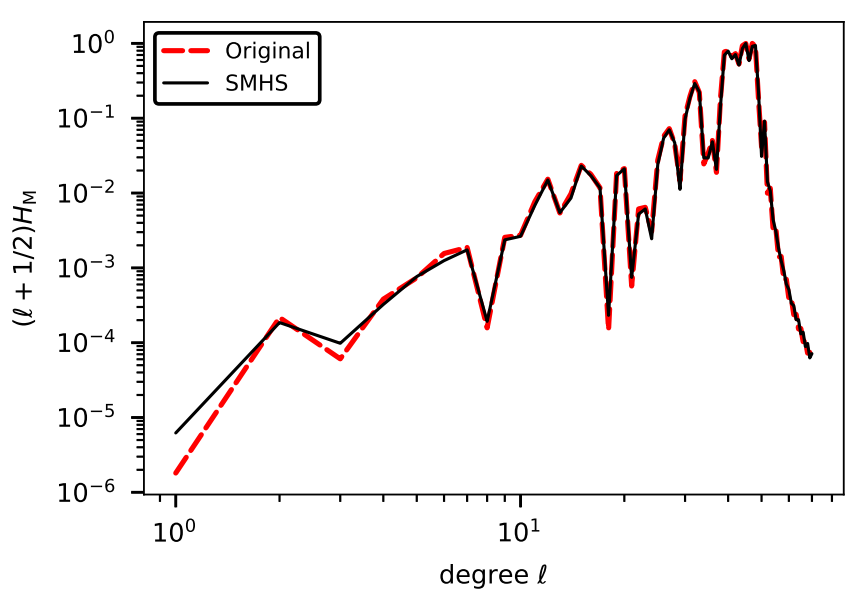

Fig. 2. Comparison of the original spectrum (red dashed) obtained using Eq. (3) for the helical magnetic field defined in Sect. 3.1 versus the spectrum obtained with Eq. (12) with only the knowledge of magnetic field. Both spectra are normalised to their maximum value.

distribution of magnetic helicity obtained from such a configuration. We apply Eq. (12) only to the magnetic field and compare the magnetic helicity spectra thus obtained with the one that we have a ready access. Figure 2 shows that it is possible to retrieve the spherical magnetic helicity spectrum (SMHS) over all scales using the angular correlation function to a very high degree.

\subsection{Magnetic helicity from simulations of a turbulent dynamo}

Another suitable test case is a simulated magnetic field from a model, where dynamo action occurs in a turbulent fluid in a spherical domain $(r, \theta, \phi)$. We employ $3 \mathrm{D}$ hydromagnetic simulations of an isothermal gas where turbulence is driven by forcing the momentum equation with a helical forcing function using the Pencil Code (Pencil Code Collaboration 2021). Along with the continuity and momentum equation, the Pencil Code solves the induction equation for $\boldsymbol{A}$,

$$
\frac{\partial \boldsymbol{A}}{\partial t}=\boldsymbol{U} \times \boldsymbol{B}-\eta \mu_{0} \boldsymbol{J}-\nabla \psi,
$$

where $\boldsymbol{U}$ is the velocity field, $\eta$ is the magnetic resistivity, $\psi$ is the electrostatic potential and $\mu_{0}$ is the magnetic permeability. This formulation ensures the solenoidality of $\boldsymbol{B}$, thus the vector potential is readily available with the Pencil Code. Here we use the resistive gauge, $\psi=-\eta \nabla \cdot \boldsymbol{A}$, for our simulations.

The simulation domain spans $0.7 R_{0} \leq r \leq R_{0}$ in radius, to mimic the convection zones of solar-like stars. Its extent in colatitude is $2 \pi / 5 \leq \theta \leq 3 \pi / 5$ and $\pi / 2$ in the azimuthal direction, hence our simulation domain is wedge shaped. We use periodic boundary conditions in the azimuthal direction. For velocity, stress-free and impenetrable boundary conditions are used for both boundaries in the radial and latitudinal direction. For the magnetic vector potential, at both latitudinal boundaries and at the bottom, perfect conductor boundary conditions are used, whereas at the top boundary a radial field condition is used. These conditions allow for magnetic helicity fluxes out of the system.

A more detailed description of the model, including the forcing function, can be found in Warnecke et al. (2011). However, there is a key difference, here the simulation domain spans $0.7 R_{0} \leq r \leq R_{0}$ in radius, and the forcing is applied at all radial locations. We limit the extent in $\theta$ and $\phi$ for computational reasons, and resolve our model with a grid of $128 \times 256 \times 256$ 

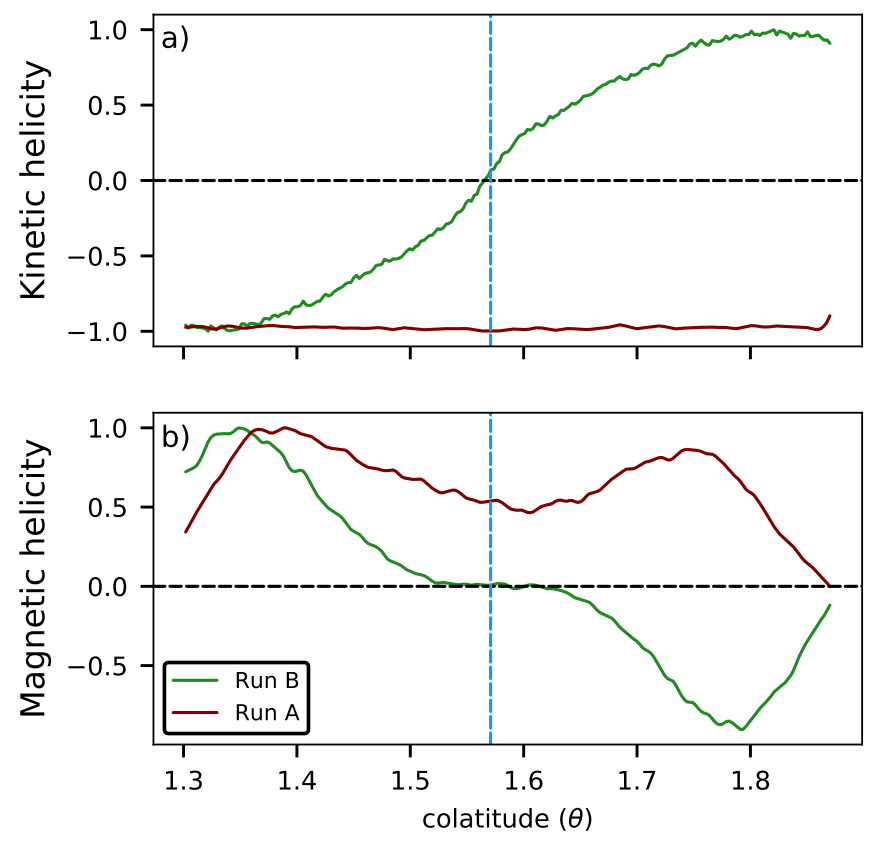

Fig. 3. Profiles of $\langle\boldsymbol{u} \cdot \boldsymbol{\omega}\rangle_{\phi t}($ panel $a)$ and $\langle\boldsymbol{A} \cdot \boldsymbol{B}\rangle_{\phi t}($ panel $b)$ as a function of $\theta$. The profiles have been normalised to their maximum value. The blue dashed line indicates the equator.

points in radius, latitude and longitude, respectively. We apply the forcing at a length scale ten times smaller than the radial extent with maximally helical forcing. We produce two different models: in Run A we keep the sign of forcing the same for both hemispheres, corresponding to a negative kinetic helicity ( $\boldsymbol{U} \cdot \boldsymbol{\omega}$ with $\boldsymbol{\omega}=\boldsymbol{\nabla} \times \boldsymbol{U}$ ) over the entire domain, while in Run $\mathrm{B}$ we vary its sign with $\cos (\theta)$, mimicking the hemispheric sign rule expected for the Sun. In both models, Runs A and B, the turbulent dynamo generated large-scale magnetic field is nonaxisymmetric.

The magnetic Reynolds number, Rm, a non-dimensional parameter quantifying the effects magnetic advection to diffusion, is 9.5 for Run A and 19.2 for Run B. The magnetic Prandtl number, $\operatorname{Pr}_{\mathrm{M}}=v / \eta$, for both runs is unity. Here $v$ is the kinematic viscosity. Figure $3 \mathrm{a}$ shows the $\phi$ - and time-averaged profiles of kinetic helicity at $r_{1} \approx 0.81 R_{0}$. For Run $\mathrm{A}$ it is negative over the entire domain, and for Run $\mathrm{B}$, it changes sign across the equator from negative to positive from northern to southern hemisphere. For such helically forced dynamos, the magnetic helicity is expected to be dominated by large-scale fields and its sign is expected to be opposite to that of kinetic helicity (Brandenburg 2001). This behaviour is reflected in our simulations as seen in Fig. $3 b$ for both Runs A and B.

At first we focus on Run A, which is homogeneously forced and thus our formalism in Sect. 2.3(a), assuming homogeneity, is applicable to it. Figure 4a shows the magnetic energy and scaled helicity spectra obtained directly from the simulation using the magnetic field and vector potential as input. We use Eqs. (2) and (3) to compute these spectra. In contrast, the energy and scaled helicity spectra shown in Fig. $4 \mathrm{~b}$ are obtained using only the magnetic field with Eqs. (11) and (12). This shows that SMHS (Fig. 4b) computed using the angular correlation function of magnetic field recovers a bihelical spectrum with positive (negative) sign at large (small) scales, which is in very good agreement with the actual helicity spectrum of the simulation (Fig. 4a). All spectra are computed at a depth of $r_{1}$ at one instance in time, and then averaged over time after the largescale field has saturated. We note that the realisability condition of $\left|H_{\mathrm{M}}\right| \leq 2 r_{1} E_{\mathrm{M}} /(\ell+1)$ (Moffatt 1978; Kahniashvili et al. $2013)$ is met. The energy spectrum shows an approximate $\ell^{-5 / 3}$ behaviour below the injection scale $(\ell \approx 42)$. Following Eq. (5), the ratio of magnetic helicity computed in real space to that of spectral space is $\approx 1.06$ which points to a reasonable agreement.

As mentioned above, this simulation was done with a choice of resistive gauge. Therefore, to test the robustness of our results against the gauge choice made, we performed an additional run identical to Run A but with a Weyl gauge, $\psi=0$. Using the Weyl gauge, the SMHS (not shown here) retrieved using the angular correlation function of magnetic field is bihelical. And as above, the ratio of helicity computed in real space to spectral space is $\approx 1.03$, thus highlighting the gauge-invariance of our approach.

For Run B, the inhomogeneous forcing results in magnetic helicity slowly changing sign as a function of colatitude at both large and small scales, where Fig. 3a reflects the opposite signs of helicity at large-scales in the two hemispheres. Such behaviour is expected in the Sun and other stars. For magnetic field extracted at $r_{1}$ from Run B, we applied the formalism presented in Sect. 2.3(b) and the spectra thus obtained are shown in Fig. 5. It indeed allows us to extract the bihelical spectrum of magnetic helicity with signs corresponding to that of the northern hemisphere, even though the helicity changes sign as a function of latitude. As an additional confirmation, following Eq. (5) we computed the ratio of helicity in real space to spectral once again, but computing the magnetic helicity in real space over the northern hemisphere only. We retrieve a value of $\approx 1.06$ from the spectrum in Fig. 5 which is again proving the accuracy of our method.

\subsection{Solar observations}

As a final test of the applicability of the formalism put forth in Sect. 2.3(b) to inhomogeneous systems, we apply it on synoptic vector magnetograms of the Sun, where a change in sign of helicity depending on the hemisphere is expected. Singh et al. (2018) studied 74 synoptic Carrington rotations (CRs) maps, using the Cartesian two-scale formalism, based on Vector Spectromagnetograph (VSM) data of the SOLIS project (Keller et al. 2003; Balasubramaniam \& Pevtsov 2011). They recovered a bihelical spectrum in a majority of cases studied. We choose here the CR 2156 of the same dataset, close the maximum of the solar cycle 24. This CR was reported to show a clear bihelical spectrum, with the signs at large and small scales following the HSR, like the majority of other CRs during cycle 24 . However, it was found to be peculiar in the sense that it shows higher power and a positive sign of helicity at intermediate scales, in comparison to the other CRs where a negative sign of helicity was prominent at these scales. Singh et al. (2018) interpreted this as a sign of the dominance of the large-scale magnetic field due to its "rejuvenation" close to the solar maximum.

Even though higher resolution data is available, we chose here to continue using SOLIS data to enable a better comparison. This way we avoid instrumental and data reduction related differences such as varying instrumental resolution and disambiguation methods needed for resolving the $180^{\circ}$ ambiguity of the transverse (perpendicular to line-of-sight) component of the magnetic field. Further details of the SOLIS synoptic map used here can be found in Singh et al. (2018).

In Fig. 6a, we reproduce Fig. 7 of Singh et al. (2018) for CR 2156 using the Cartesian two-scale formalism, that is Eqs. (8) and (9). In panel $b$ of the same figure, we show the 

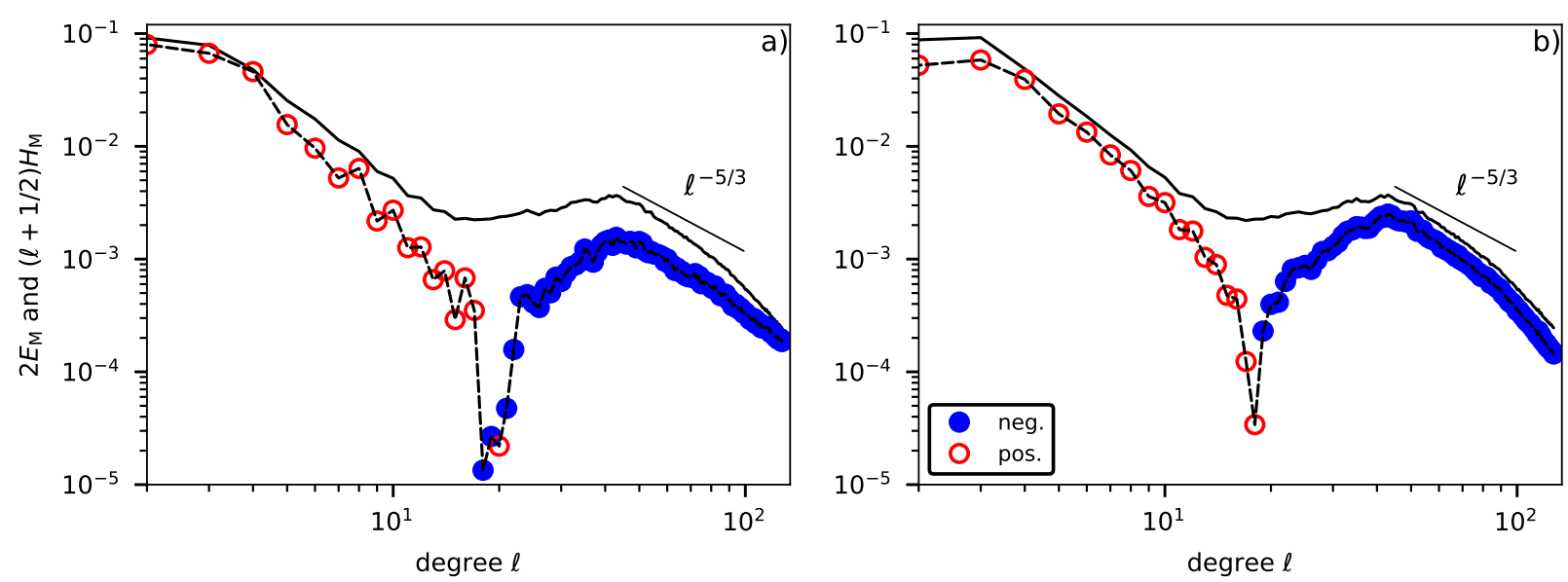

Fig. 4. Magnetic energy (solid) and scaled helicity spectra (dashed) obtained at a depth of $r_{1}$ for Run A. Panel a: the energy and helicity spectra are obtained using the magnetic field and vector potential directly from the simulation data, whereas in panel $b$, we use the angular correlation function given by Eq. (10) to evaluate the energy spectra and the SMHS using only the magnetic field data.

magnetic energy and SMHS of the same CR, computed using Eq. (11) and the steps described in Sect. 2.3(b) respectively. The spectra shown in these two panels are in very good agreement and reveal the bihelical nature of the solar magnetic field. The magnetic helicity for this CR peaks at $209-300 \mathrm{Mm}$ which is an even larger scale than the $160 \mathrm{Mm}$ reported by Singh et al. (2018). This lends support to their rejuvenation argument. We note, however, that SMHS recovers somewhat less power and energy at the largest scales than the Cartesian approach, and, consequently, the spectral slope corresponding to lower $k$ is steeper in the SMHS case, and no longer clearly consistent with the Kazantsev scaling, $k^{3 / 2}$. At the higher wavenumbers, SMHS shows more power and energy and hence a less steep spectral slope is observed than in the Cartesian approach.

A contrasting interpretation of the same CR is reported by Pipin et al. (2019), where they find this CR to be a violation of HSR. This discrepancy is likely a result of the different definition of scales. They define small and large scales purely based on azimuthal averaging and report the small-scale magnetic helicity density of this CR in the southern hemisphere to be negative. The latter is mainly attributed to the presence of a prominent active region (NOAA 12192) with negative helicity in that hemisphere (see Fig. 3 of Pipin et al. 2019). Their definition of small-scale magnetic helicity density also includes large-scale non-axisymmetric contributions. We regard this definition as a possible source for the dissimilar interpretations. With our approach, we demonstrated the possibility to reliably retrieve magnetic helicity spectra for large-scale non-axisymmetric magnetic fields in Sect. 3.2. For this particular CR, the presence of AR 12192 is seen as increased power at intermediate to large scales for the SMHS (Fig. 6b), with the sign in agreement with the low- $k$ (large scale) part of the helicity spectrum. This highlights that categorising a CR as a violation of HSR or not is a delicate issue. SMHS offers a much richer picture, and hence can be regarded as a better-suited tool for such classification.

\section{Discussion}

Our aim with this study was to extend the Cartesian formalism for inferring spectral distributions of magnetic energy and helicity using the two-point correlation tensor to spherical geometry. The need for this was alluded to in Brandenburg (2018), since the analysis of Brandenburg et al. (2017) maps the magnetic field

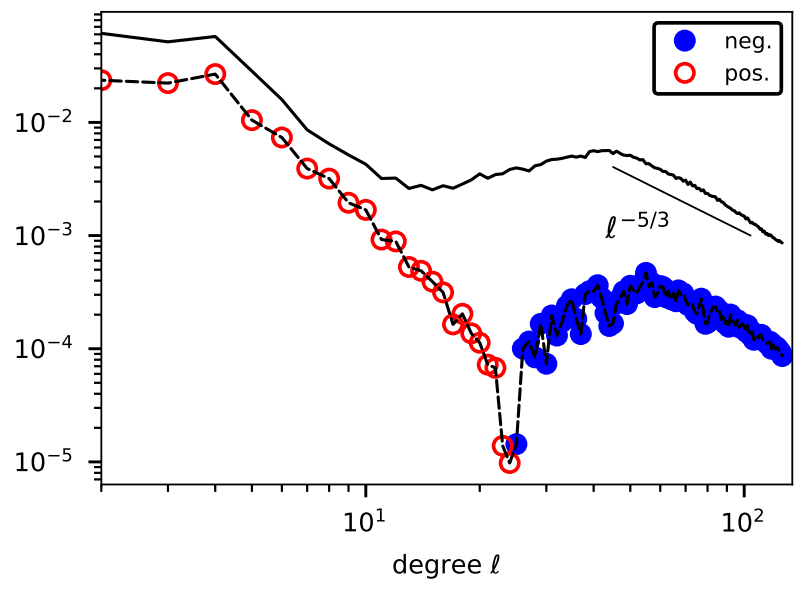

Fig. 5. Magnetic energy (solid) and scaled helicity spectra (dashed) for Run B. For the energy spectrum, we continue to use Eqs. (10) and (11), whereas the SMHS in this case is obtained by the inhomogeneous formulation discussed in Sect. 2.3(b) (see Appendix C for a derivation).

vector data from solar observations to a 2D Cartesian surface. Apart from loosening this restriction, going to spherical geometry additionally allows us to infer helicity spectra directly from full disk magnetograms, instead of waiting for the build-up of a synoptic map for one Carrington rotation. Analogously, using ZDI based magnetic field observations (for example Vidotto 2016) such spectra can also be retrieved for other stars limiting to lower spherical harmonic degree, similar to the study of Lund et al. (2020) who reported average magnetic helicity density using stellar ZDI data. It also enables comparison with stateof-the-art dynamo models of the Sun or stars, which mostly solve the induction equation for $\boldsymbol{B}$. Using our formalism one can readily compute magnetic helicity spectra without explicitly computing $\boldsymbol{A}$. For most of the above mentioned observations and models, a change in sign of kinetic and magnetic helicity across the equator, owing to stratification and rotation breaking reflectional symmetry, is expected. Our formalism in Sect. 2.3(b) (also suggested by Brandenburg 2019) by correlating fields at spherical harmonic degrees shifted by one, is particularly suited for such cases. Our tests in Sects. 3.2 and 3.3 confirm its applicability to inhomogeneous systems. Thus we can confirm and extend 

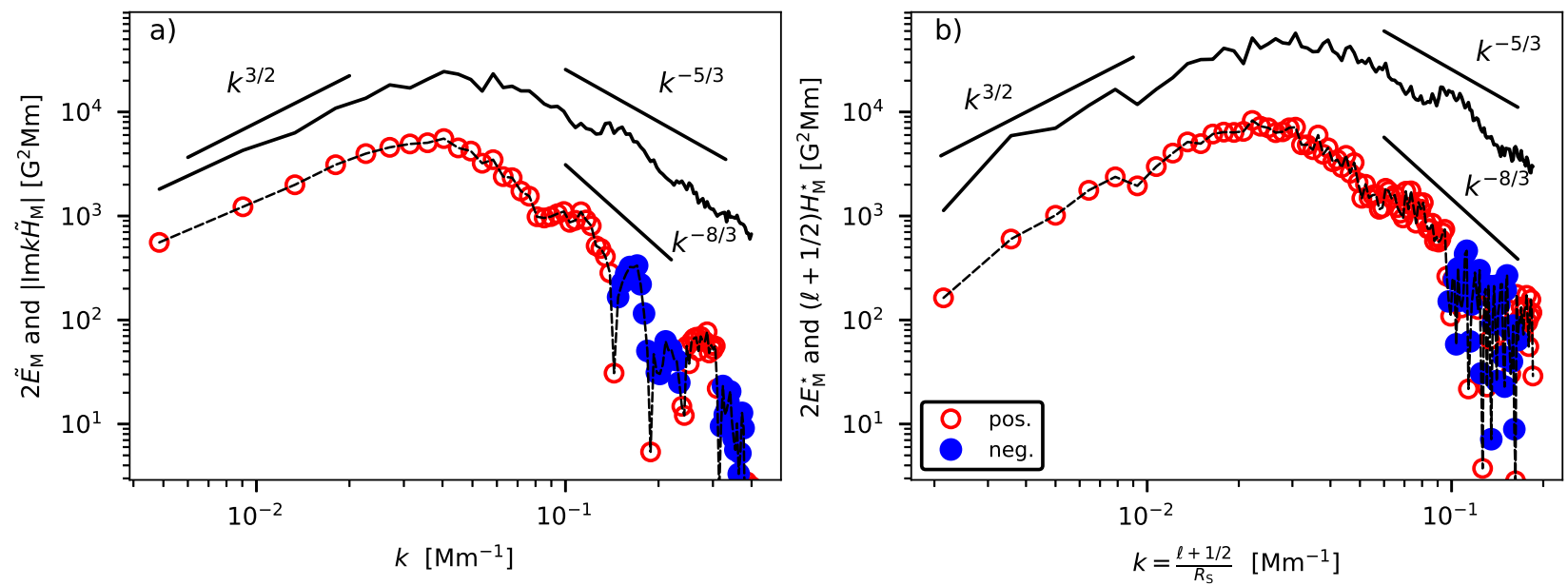

Fig. 6. Magnetic energy (solid) and scaled helicity (dashed) spectra for CR 2156 obtained using SOLIS/VSM data. Panel a: we have reproduced Fig. 7 of Singh et al. (2018) using. Panel $b$ : magnetic and SHMS obtained using the angular correlation function in spherical geometry, see Sect. 2.3. Both plots are shown as a function of wavenumber using the Jeans relation $k R_{\mathrm{S}} \approx \ell+1 / 2$, where $R_{\mathrm{S}}$ is the solar radius taken to be approximately $700 \mathrm{Mm}$. To enable a better comparison between the figures we have plotted $E_{\mathrm{M}}^{\star}(\ell)=R_{\mathrm{S}} E_{\mathrm{M}}(\ell)$ and $H_{\mathrm{M}}^{\star}(\ell)=R_{\mathrm{S}} H_{\mathrm{M}}(\ell)$ in panel $b$.

findings of previous studies focusing on the HSR, reported, e.g., in Singh et al. (2018), Pipin et al. (2019).

Cross-helicity is also expected to play a role in generating large-scale magnetic fields (Yokoi 2013). Previous studies have determined cross-helicity from observations (Zhang \& Brandenburg 2018, and references therein) mostly with the line-of-sight component of the velocity field. However, information about the full velocity field vector can also be obtained for a significant portion of the full disk as demonstrated in Rincon et al. (2017). This velocity field vector can be used with our method to infer cross-helicity spectra $(\boldsymbol{u} \cdot \boldsymbol{b})$.

\section{Conclusions}

In order to investigate the mechanisms responsible for largescale magnetic fields present in the Sun and other stars, having a knowledge of magnetic helicity and specifically its distribution over scales is important. In this study, we demonstrate an extension of an existing Cartesian formalism, relying on the two-point correlation tensor of the magnetic field, to spherical geometry. This allows us access to magnetic helicity spectra by appealing to a more fundamental definition of helicity in terms of the linkage of the magnetic field lines, and circumvents the need to compute the magnetic vector potential. We tested this approach on a variety of illustrative examples before demonstrating its application to the solar vector synoptic maps. This enables an extensive analysis of different datasets from different instruments, to vet the robustness of the bihelical nature of solar magnetic field against instrumental effects.

Our approach naturally captures the bihelicity of the magnetic field in each hemisphere, and also the slow modulation of the helicity as a function of latitude. This is relevant for the Sun, where magnetic helicity on both large and small scales is expected to change sign across the equator. The true nature of the helicity distribution over the whole sphere is revealed, and a potential contamination of power and sign of the helicity from both hemispheres is avoided. Such a contamination could easily lead to an apparent violation of the HSR even when the rule is obeyed. The method discussed here remedies this, and it is expected to find applications in systems involving such rich distribution of magnetic helicity.
Acknowledgements. MJK acknowledges the support of the Academy of Finland ReSoLVE Centre of Excellence (grant No. 307411). AP was funded by the International Max Planck Research School for Solar System Science at the University of Göttingen. This project has received funding from the European Research Council under the European Union's Horizon 2020 research and innovation programme (project "UniSDyn", grant agreement n:o 818665). SOLIS data used here are produced cooperatively by NSF/NSO and NASA/LWS.

\section{References}

Balasubramaniam, K. S., \& Pevtsov, A. 2011, in Solar Physics and Space Weather Instrumentation IV, eds. S. Fineschi, \& J. Fennelly, SPIE Conf. Ser., 8148,814809

Bao, S. D., Zhang, H. Q., Ai, G. X., \& Zhang, M. 1999, A\&AS, 139, 311

Berger, M. A. 1984, Geophys. Astrophys. Fluid Dyn., 30, 79

Berger, M. A., \& Field, G. B. 1984, J. Fluid Mech., 147, 133

Brandenburg, A. 2001, ApJ, 550, 824

Brandenburg, A. 2018, J. Plasma Phys., 84, 735840404

Brandenburg, A. 2019, ApJ, 883, 119

Brandenburg, A., \& Subramanian, K. 2005, Phys. Rep., 417, 1

Brandenburg, A., Petrie, G. J. D., \& Singh, N. K. 2017, ApJ, 836, 21

Chandrasekhar, S. 1961, Hydrodynamic and Hydromagnetic Stability (Oxford: Clarendon Press)

Chandrasekhar, S., \& Kendall, P. C. 1957, ApJ, 126, 457

Finn, J., \& Antonsen, T., Jr. 1985, Plasma Phys. Control. Fusion, 9, 111

Hawkes, G., \& Berger, M. A. 2018, Sol. Phys., 293, 109

Ji, H. 1999, Phys. Rev. Lett., 83, 3198

Kahniashvili, T., Tevzadze, A. G., Brandenburg, A., \& Neronov, A. 2013, Phys. Rev. D, 87, 083007

Keller, C. U., Harvey, J. W., \& Giampapa, M. S. 2003, in Innovative Telescopes and Instrumentation for Solar Astrophysics, eds. S. L. Keil, \& S. V. Avakyan, SPIE Conf. Ser., 4853, 194

Lund, K., Jardine, M., Lehmann, L. T., et al. 2020, MNRAS, 493, 1003

Moffatt, H. K. 1969, J. Fluid Mech., 35, 117

Moffatt, H. K. 1978, Magnetic Field Generation in Electrically Conducting Fluids (Cambridge: Cambridge University Press)

Moffatt, H. K., \& Ricca, R. L. 1992, Proc. R. Soc. London Ser. A, 439, 411

Pariat, E., Leake, J. E., Valori, G., et al. 2017, A\&A, 601, A125

Peebles, P. J. E. 1973, ApJ, 185, 413

Pencil Code Collaboration (Brandenburg, A., et al.) 2021, J. Open Sour. Softw., 6, 2807

Pevtsov, A. A., Canfield, R. C., \& Metcalf, T. R. 1995, ApJ, 440, L109

Pipin, V. V., Pevtsov, A. A., Liu, Y., \& Kosovichev, A. G. 2019, ApJ, 877, L36

Prior, C., Hawkes, G., \& Berger, M. A. 2020, A\&A, 635, A95

Rincon, F. 2019, J. Plasma Phys., 85, 205850401

Rincon, F., Roudier, T., Schekochihin, A. A., \& Rieutord, M. 2017, A\&A, 599, A69

Roberts, P. H., \& Soward, A. M. 1975, Astron. Nachr., 296, 49

Schaeffer, N. 2013, Geochem. Geophys. Geosyst., 14, 751

Seehafer, N. 1990, Sol. Phys., 125, 219 
A\&A 654, A3 (2021)

Seehafer, N. 1996, Phys. Rev. E, 53, 1283

Semel, M. 1989, A\&A, 225, 456

Singh, N. K., Käpylä, M. J., Brandenburg, A., et al. 2018, ApJ, 863, 182

Subramanian, K., \& Brandenburg, A. 2006, ApJ, 648, L71

Sullivan, C. B., \& Kaszynski, A. 2019, J. Open Sour. Softw., 4, 1450

Thalmann, J. K., Moraitis, K., Linan, L., et al. 2019, ApJ, 887, 64

Valori, G., Pariat, E., Anfinogentov, S., et al. 2016, Space Sci. Rev., 201, 147

Vidotto, A. A. 2016, MNRAS, 459, 1533

Warnecke, J., \& Peter, H. 2019, A\&A, submitted [arXiv:1910. 06896]

Warnecke, J., Brandenburg, A., \& Mitra, D. 2011, A\&A, 534, A11
Wieczorek, M. A., \& Meschede, M. 2018, Geochem. Geophys. Geosyst., 19, 2574

Woltjer, L. 1958, Proc. Natl. Acad. Sci., 44, 833

Yokoi, N. 2013, Geophys. Astrophys. Fluid Dyn., 107, 114

Yousef, T. A., \& Brandenburg, A. 2003, A\&A, 407, 7

Zhang, H., \& Brandenburg, A. 2018, ApJ, 862, L17

Zhang, H., Sakurai, T., Pevtsov, A., et al. 2010, MNRAS, 402, L30

Zhang, H., Brandenburg, A., \& Sokoloff, D. D. 2014, ApJ, 784, L45

Zhang, H., Brandenburg, A., \& Sokoloff, D. D. 2016, ApJ, 819, 146 


\section{Appendix A: Spherical correlation function}

Here we show a brief derivation of how the magnetic energy and magnetic helicity spectra can be extracted from the twopoint angular correlation function. We note that we are interested to determine these spectra from the measurements of vector magnetic field from the surface of a sphere, e.g., the Sun. We may write the following expression for the two-point angular correlation function under the homogeneous conditions (Peebles 1973):

$M_{i j}(\chi)=\frac{\iint B_{i}\left(\mathbf{\Omega}_{1}\right) B_{j}\left(\mathbf{\Omega}_{2}\right) \delta\left(\cos \chi_{12}-\cos \chi\right) \mathrm{d} \boldsymbol{\Omega}_{1} \mathrm{~d} \boldsymbol{\Omega}_{2}}{\iint \delta\left(\cos \chi_{12}-\cos \chi\right) \mathrm{d} \boldsymbol{\Omega}_{1} \mathrm{~d} \boldsymbol{\Omega}_{2}}$,

where $\chi_{12}$ is the angle between the directions $\boldsymbol{\Omega}_{1}=\boldsymbol{\Omega}_{1}\left(\theta_{1}, \phi_{1}\right)$ and $\boldsymbol{\Omega}_{2}=\boldsymbol{\Omega}_{2}\left(\theta_{2}, \phi_{2}\right)$ which are the position vectors of the two points on the spherical surface; $\theta$ 's and $\phi$ 's represent the colatitude and azimuth, respectively. The homogeneity is ensured by the delta function in Eq. (A.1) where the correlation function depends only on the angular separation $\chi$, and the angular integrals yield an average over the sphere. The normalisation in the denominator may be determined by expanding the delta function in Eq. (A.1) in terms of the Legendre Polynomials as

$\delta\left(\cos \chi_{12}-\cos \chi\right)=\sum_{\ell} a_{\ell} P_{\ell}\left(\cos \chi_{12}\right)$.

Writing the orthonormality condition for $P_{\ell}$ as

$\int_{-1}^{1} P_{\ell}(\cos \alpha) P_{\ell^{\prime}}(\cos \alpha) \mathrm{d} \cos \alpha=\frac{2 \delta_{\ell \ell^{\prime}}}{2 \ell+1}$,

the coefficients $a_{\ell}$ are found to be

$a_{\ell}=\frac{2 \ell+1}{2} P_{\ell}(\cos \chi)$,

giving

$\delta\left(\cos \chi_{12}-\cos \chi\right)=\sum_{\ell} \frac{2 \ell+1}{2} P_{\ell}(\cos \chi) P_{\ell}\left(\cos \chi_{12}\right)$.

By expanding $P_{\ell}\left(\cos \chi_{12}\right)$ in terms of the spherical harmonics as

$P_{\ell}\left(\cos \chi_{12}\right)=\frac{4 \pi}{2 \ell+1} \sum_{m=-\ell}^{\ell} Y_{\ell}^{m}\left(\mathbf{\Omega}_{1}\right) Y_{\ell}^{m *}\left(\mathbf{\Omega}_{2}\right)$,

we find, after straightforward algebra, that

$\iint \delta\left(\cos \chi_{12}-\cos \chi\right) \mathrm{d} \boldsymbol{\Omega}_{1} \mathrm{~d} \boldsymbol{\Omega}_{2}=8 \pi^{2}$

for which, we have used $\sqrt{4 \pi} Y_{0}^{0}=1, \int Y_{\ell}^{m}(\mathbf{\Omega}) \mathrm{d} \boldsymbol{\Omega}=\sqrt{4 \pi} \delta_{\ell 0} \delta_{m 0}$ and $P_{0}(\cos \chi)=1$. This gives Eq. (10).

\section{Appendix B: Magnetic energy spectrum}

Analogous to the Fourier case, we can define the magnetic energy spectrum, which gives a distribution of the magnetic energy over $\ell$, as

$2 E_{\mathrm{M}}(\ell)=2 \pi(2 \ell+1) \int_{-1}^{1} \delta_{i j} M_{i j}(\chi) P_{\ell}(\cos \chi) \mathrm{d} \cos \chi$,

which essentially depends on the trace of the two-point function, $\operatorname{Tr}\left[M_{i j}(\chi)\right]=\delta_{i j} M_{i j}$. Equation (B.1) may be understood by first expanding this trace as $\delta_{i j} M_{i j}(\chi)=\sum_{\ell} c_{\ell} P_{\ell}(\cos \chi)$, and then determining the coefficient $c_{\ell}$ in a standard way, which gives

$c_{\ell}=\frac{2 \ell+1}{2} \int_{-1}^{1} \delta_{i j} M_{i j}(\chi) P_{\ell}(\cos \chi) \mathrm{d} \cos \chi$

By defining the magnetic energy spectrum, $E_{\mathrm{M}}(\ell)$, in terms of $c_{\ell}$ as $2 E_{\mathrm{M}}(\ell)=4 \pi c_{\ell}$, we arrive at Eq. (B.1) which, after using Eq. (10) together with Eqs. (A.5), (A.6), and (A.3), gives the following simple expression for the energy spectrum:

$2 E_{\mathrm{M}}(\ell)=\sum_{m=-\ell}^{\ell}\left(b_{r}^{\ell m}\right)^{2}+\left(b_{\theta}^{\ell m}\right)^{2}+\left(b_{\phi}^{\ell m}\right)^{2}$,

where $b_{i}^{\ell m}=\int B_{i}(\mathbf{\Omega}) Y_{\ell}^{m *}(\mathbf{\Omega}) \mathrm{d} \mathbf{\Omega}$ is the expansion coefficient of the $i^{\text {th }}$ component of the magnetic field $\boldsymbol{B}$.

\section{Appendix C: Magnetic helicity spectrum}

The Gauss linking formula yields the magnetic helicity directly in terms of the magnetic field $\boldsymbol{B}(\boldsymbol{x})$ by determining the fluxlinkages:

$\mathcal{H}_{\mathrm{M}}=\frac{1}{4 \pi} \iint \frac{\boldsymbol{x}-\boldsymbol{y}}{|\boldsymbol{x}-\boldsymbol{y}|^{3}} \cdot[\boldsymbol{B}(\boldsymbol{x}) \times \boldsymbol{B}(\boldsymbol{y})] \mathrm{d}^{3} x \mathrm{~d}^{3} y$.

Here $\boldsymbol{x}$ and $\boldsymbol{y}$ are the position vectors of two points on the surface of a sphere. Using

$\frac{\boldsymbol{x}-\boldsymbol{y}}{|\boldsymbol{x}-\boldsymbol{y}|^{3}}=\boldsymbol{\nabla}_{y} \frac{1}{|\boldsymbol{x}-\boldsymbol{y}|}, \quad$ and $\quad \frac{1}{|\boldsymbol{x}-\boldsymbol{y}|}=\frac{1}{R} \sum_{\ell=0}^{\infty} P_{\ell}(\cos \chi)$,

with $R, \chi$, and $P_{\ell}$ being, respectively, the radius of the sphere, the angle between $\boldsymbol{x}$ and $\boldsymbol{y}$, and the Legendre polynomial of degree $\ell$, we rewrite the expression for the magnetic helicity,

$\mathcal{H}_{\mathrm{M}}=\sum_{\ell=0}^{\infty} H_{M}(\ell)$

where

$$
\begin{gathered}
H_{M}(\ell)=\frac{1}{2 \ell+1} \iint \boldsymbol{\nabla}^{\prime}\left(\sum_{m=-\ell}^{\ell} Y_{\ell}^{m}(\mathbf{\Omega}) Y_{\ell}^{m *}\left(\boldsymbol{\Omega}^{\prime}\right)\right) . \\
\cdot\left[\boldsymbol{B}(\mathbf{\Omega}) \times \boldsymbol{B}\left(\mathbf{\Omega}^{\prime}\right)\right] \mathrm{d} \mathbf{\Omega} \mathrm{d} \mathbf{\Omega}^{\prime}
\end{gathered}
$$

for which, we assumed a unit sphere $(R=1)$ and expanded the Legendre polynomials in terms of the spherical harmonics as

$P_{\ell}(\cos \chi)=\frac{4 \pi}{2 \ell+1} \sum_{m=-\ell}^{\ell} Y_{\ell}^{m}(\theta, \phi) Y_{\ell}^{m *}\left(\theta^{\prime}, \phi^{\prime}\right)$.

Directions $\boldsymbol{\Omega}=\boldsymbol{\Omega}(\theta, \phi)$ and $\boldsymbol{\Omega}^{\prime}=\boldsymbol{\Omega}^{\prime}\left(\theta^{\prime}, \phi^{\prime}\right)$ correspond to the position vectors $\boldsymbol{x}$ and $\boldsymbol{y}$, respectively, with $\mathrm{d} \boldsymbol{\Omega}=\sin \theta \mathrm{d} \theta \mathrm{d} \phi$ and $\mathrm{d} \boldsymbol{\Omega}^{\prime}=\sin \theta^{\prime} \mathrm{d} \theta^{\prime} \mathrm{d} \phi^{\prime}$. Employing the Jeans relation which expresses the wavenumber $k$ on the surface of the sphere of radius $R$ (assumed as unity here) in terms of the spherical harmonic degree $\ell$ by $k R=\sqrt{\ell(\ell+1)} \approx \ell+1 / 2$, we can write

$k H_{M}(\ell)=(\ell+1 / 2) H_{M}(\ell)$.

Thus the scaled magnetic helicity spectrum, $\mathrm{kH}_{M}(\ell)$, which has the same dimensions as for the magnetic energy spectrum, $E_{M}(\ell)$, may be determined by using equations (C.4) and (C.6). 
It is important to note that Eq. (C.4) provides a general expression for the magnetic helicity, and thus, in its current form, it does not make any assumption of homogeneity. This same equation may be used for both homogeneous and inhomogeneous cases by suitably writing the two-point function $M_{i j}$ which naturally appears when we take an ensemble average of Eq. (C.4). We take (i) $M_{i j}=M_{i j}(\chi)$ for homogeneous case in which it depends only on the angular separation $(\chi)$ between the two points, and (ii) $M_{i j}=M_{i j}(\boldsymbol{\Omega}, \chi)$ for inhomogeneous case where $M_{i j}$ depends also on the position $(\boldsymbol{\Omega})$ on the surface of the sphere. In weakly inhomogeneous turbulence, which appears to be more relevant in the solar context, $M_{i j}$ is expected to vary rapidly with $\chi$ while showing a slow variation with position $\boldsymbol{\Omega}$ on the sphere. Here we are more interested in only the latitudinal variation which involves a sign change of magnetic helicity across the equator, simultaneously at both, small and large, length scales.

To numerically evaluate the integrals in Eq. (C.4) we use the vectorial generalisation of spherical harmonics implemented in the SHTns library (Schaeffer 2013). 